\title{
Accounting for center-of-mass target motion using convolution methods in Monte Carlo-based dose calculations of the lung
}

\author{
Indrin J. Chetty, ${ }^{\text {a) }}$ Mihaela Rosu, Daniel L. McShan, Benedick A. Fraass, \\ James M. Balter, and Randall K. Ten Haken \\ Department of Radiation Oncology, The University of Michigan Ann Arbor, Michigan 48109-0010
}

(Received 10 November 2003; revised 30 December 2003; accepted for publication 22 January

2004; published 25 March 2004)

\begin{abstract}
We have applied convolution methods to account for some of the effects of respiratory induced motion in clinical treatment planning of the lung. The 3-D displacement of the GTV center-of-mass $(\mathrm{COM})$ as determined from breath-hold exhale and inhale CT scans was used to approximate the breathing induced motion. The time-course of the GTV-COM was estimated using a probability distribution function (PDF) previously derived from diaphragmatic motion [Med. Phys. 26, 715720 (1990)] but also used by others for treatment planning in the lung [Int. J. Radiat. Oncol., Biol., Phys. 53, 822-834 (2002); Med. Phys. 30, 1086-1095 (2003)]. We have implemented fluence and dose convolution methods within a Monte Carlo based dose calculation system with the intent of comparing these approaches for planning in the lung. All treatment plans in this study have been calculated with Monte Carlo using the breath-hold exhale CT data sets. An analysis of treatment plans for 3 patients showed substantial differences (hot and cold spots consistently greater than $\pm 15 \%$ ) between the motion convolved and static treatment plans. As fluence convolution accounts for the spatial variance of the dose distribution in the presence of tissue inhomogeneities, the doses were approximately 5\% greater than those calculated with dose convolution in the vicinity of the lung. DVH differences between the static, fluence and dose convolved distributions for the CTV were relatively small, however, larger differences were observed for the PTV. An investigation of the effect of the breathing PDF asymmetry on the motion convolved dose distributions showed that reducing the asymmetry resulted in increased hot and cold spots in the motion convolved distributions relative to the static cases. In particular, changing from an asymmetric breathing function to one that is symmetric results in an increase in the hot/cold spots of $\pm 15 \%$ relative to the static plan. This increase is not unexpected considering that the target spends relatively more time at inhale as the asymmetry decreases (note that the treatment plans were generated using the exhale CT scans). (C) 2004 American Association of Physicists in Medicine. [DOI: 10.1118/1.1669083]
\end{abstract}

Key words: Monte Carlo, lung GTV, center-of-mass breathing motion, fluence convolution, dose convolution

\section{INTRODUCTION}

Conformal radiotherapy treatment planning is typically based on a single CT scan, which represents one instance of the patient anatomy. However, in order to more accurately estimate the dose to the tumor, it is important in such sites as the lung and liver, that the respiratory-induced motion be accounted for within the dose calculations. Differences that result between the planned dose distributions in the static and motion-compensated cases can be clinically significant (particularly in the context of dose escalation), as pointed out in a recent study by Rosu et al., involving treatment planning of tumors in the liver. Several investigators have proposed methods to account for organ motion in treatment planning. ${ }^{1-7,11}$ One traditional approach has been to convolve the static dose distributions with functions that approximate the breathing. ${ }^{1,7}$ In particular, the application of this approach for treatment planning in the liver by Lujan et al. ${ }^{1}$ has shown that applying a single convolution to the static dose distribution is sufficient to predict the dose distribution for the given fractionated treatment; intra-fraction effects were found to average out over the course of many fractions. Potential limitations ${ }^{1,8,9}$ of the dose convolution approach include the following assumptions: (a) the dose is spatially invariant for small changes in the geometry (i.e., the convolution of the dose is conducted in an assumed homogeneous medium), (b) the motion is based on a rigid body approximation, and (c) the method applies over an "infinite" number of fractions.

More recently, the use of Monte Carlo based dose calculation algorithms have facilitated a new approach to account for random setup errors ${ }^{10}$ and breathing-induced motion. ${ }^{11}$ This approach termed "fluence convolution" is performed by convolving the particle fluence with the appropriate motionrelated functions. Fluence convolution is based on the reciprocity principle of motion between the incident fluence and the patient-convolving the fluence with the patient anatomy held fixed is theoretically equivalent to shifting patient anatomy with the fluence being stationary. ${ }^{10}$ The benefit of fluence convolution over dose convolution is that it is based on a direct simulation approach, that is, the dose is recalcu- 
lated at each instance of the translated fluence. The calculated dose distribution is therefore not spatially invariant as in the case of dose convolution. However, as with dose convolution, fluence convolution is also limited by the rigid body approximation and by the fact that dose fraction effects are ignored.

The intent of this study was to apply convolution methods (both fluence and dose convolution) to account for some of the effects of respiratory-induced motion of the gross tumor volume (GTV) in treatment planning for lesions located within the lung. Motion of the GTV has been estimated by evaluating the excursion of the center-of-mass (COM) between normal breath-hold exhale and inhale CT scans. We compare calculations using the convolution implementations with those from the static (no motion case) for the CTV and PTV. In all cases, treatment plans have been generated using a Monte Carlo based dose calculation algorithm.

\section{METHODS AND MATERIALS}

\section{A. Image acquisition and target center-of-mass motion}

As part of a new CT imaging study protocol (at our institution) for patients with lung cancer, CT data are acquired in the normal breath-hold inhale and exhale positions, as well as at an arbitrary free-breathing state. In this study, the breath-hold inhale and exhale CT scans were used to estimate the center-of-mass (COM) motion of the gross tumor volume (GTV). The GTV was delineated by the physician on the inhale and exhale CT data sets. The treatment planning volumes: GTV, clinical target volume (CTV), and planning target volume (PTV) are those recommended by the ICRU Report No. 50. ${ }^{12}$ During standard (static) treatment planning, the GTV is outlined on the breath-hold exhale CT scan; the CTV is formed by a uniform, $0.5 \mathrm{~cm}$ expansion of the GTV and the PTV includes a further $1.0 \mathrm{~cm}$ uniform expansion for setup uncertainties and breathing-induced motion of the tumor.

The general methodology for assessing target motion was to calculate the displacement of the GTV-COM between inhale and exhale extents of breathing. This was accomplished by calculating the COM coordinates on the inhale and exhale CT scans using the equation

$$
x_{\mathrm{com}}=\frac{\sum_{i} x_{i} m_{i}}{\sum_{i} m_{i}}, \quad y_{\mathrm{com}}=\frac{\sum_{i} y_{i} m_{i}}{\sum_{i} m_{i}}, \quad z_{\mathrm{com}}=\frac{\sum_{i} z_{i} m_{i}}{\sum_{i} m_{i}},
$$

where $x_{i}, y_{i}, z_{i}$ represent the coordinates, and $m_{i}$ the mass of voxel $i$. Voxels for the COM calculation constitute the 3-D generated GTV volume within the treatment planning system (UMPlan). The displacement vector of the GTVCOM $\left(R_{\text {com }}\right)$ between inhale and exhale is then given by the equation

$$
R_{\text {com }}(x, y, z)=\left(x_{I}-x_{E}\right) \hat{i}+\left(y_{I}-y_{E}\right) \hat{j}+\left(z_{I}-z_{E}\right) \hat{k},
$$

where the subscripts $I$ represent the COM coordinates in the inhale position and the subscripts $E$ represent those in the exhale position.

\section{B. Monte Carlo virtual source model and the convolution implementations}

\section{Monte Carlo virtual source model}

A virtual source model ${ }^{13,14}$ has been developed for Monte Carlo dose calculations using the Dose Planning Method

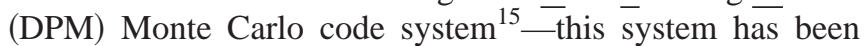
integrated into our in-house treatment planning system, UMPlan (University of Michigan, Ann Arbor, MI). The source model was reconstructed from phase space calculations of the treatment head components of a Varian 21EX linear accelerator (Varian Associates, Palo Alto, CA) generated with the BEAMnrc Monte Carlo code (CNRC, Ottawa, CN). The source particle's position and energy are sampled from the respective fluence and bremsstrahlung distributions, and the direction is calculated assuming that the particle emerged from a point. Arbitrary field shapes are simulated by multiplying the uncollimated fluence map by a matrix describing the MLC configuration. In order to account for the finite width of the target and leaf edge penumbral effects, the shaped-beam fluence map is convolved with a Gaussian kernel as described previously. ${ }^{13}$

\section{Fluence convolution and motion of the COM}

The general fluence convolution method involves convolving the static beam fluence with a function that describes the respiratory-induced motion. In this paper we use the following notation: $\Phi_{\text {static }}$ represents the MLC-shaped field static fluence distribution, $F_{\text {motion }}$ the function describing the breathing motion, and $\Phi_{\text {motion }}$ the convolved fluence map which incorporates the motion. For a point, $r$, that undergoes the motion, we have

$$
\begin{aligned}
\Phi_{\text {motion }}(r) & =F_{\text {motion }} \otimes \Phi_{\text {static }} \\
& =\int_{r^{\prime}} F_{\text {motion }}\left(r-r^{\prime}\right) \Phi_{\text {static }}\left(r^{\prime}\right) d r^{\prime} .
\end{aligned}
$$

$F_{\text {motion }}$, is the probability distribution function (PDF) derived from the position-time function for tumor motion, and may be estimated by observing the breathing-induced motion under fluoroscopy as described previously by other investigators. ${ }^{2,3,16,17}$ To estimate the GTV-COM position as a function of time we use a function originally proposed by Lujan et al. ${ }^{1}$ to account for breathing-induced (superiorinferior) motion in treatment planning of lesions in the liver. This function is given by

$$
\mathrm{z}(\mathrm{t})=z_{0}-a \cos ^{2 n}(\pi t / \tau-\phi),
$$

where $z_{0}$ is the position at exhale, $a$ the amplitude of the motion, $\tau$ the period of the breathing cycle, $n$ a parameter that determines the degree of asymmetry of the model (i.e., how much the respiratory cycle is biased toward exhale), and $\phi$ the starting phase of the breathing cycle. ${ }^{1}$ In this study we assume that the GTV-COM moves according to the function in Eq. (4) along a trajectory $R_{\text {com }}(x, y, z)$. Lujan et al. ${ }^{1}$ show further that Eq. (4) can be recast to yield a probability that a point lies between $z$ and $z+d z$, which is equal to the fraction 


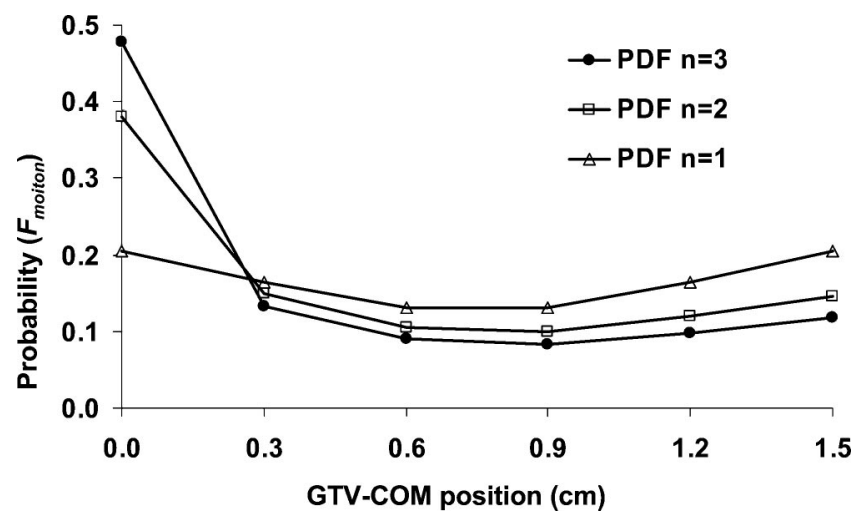

FIG. 1. Probability as a function of amplitude of the GTV center-of-mass $(\mathrm{COM})$ motion as represented in Eq. (5). Plots are shown for functions with varying degrees of asymmetry, $n=1-3$. The amplitude of motion is $1.5 \mathrm{~cm}$.

of the total time that a point spends in the interval between $t$ and $t+d t$. The resulting PDF (labeled $F_{\text {motion }}$ ), upon replacing $z$ with $r$ is as follows:

$$
\begin{aligned}
F_{\text {motion }}(r)= & \left\{n a \pi\left(\frac{R_{0}-r}{a}\right)^{(2 \mathrm{n}-1) / 2 \mathrm{n}}\right. \\
& \left.\times\left[1-\left(\frac{R_{0}-r}{a}\right)^{1 / n}\right]^{1 / 2}\right\}^{-1},
\end{aligned}
$$$$
\text { for } R_{0}-a<r<R_{0} \text {, }
$$

where $R_{0}$ represents the position of the GTV-COM at exhale, and $r$ the arbitrary position along the trajectory $R_{\text {com }}(x, y, z)$. The assumption here is that the 3-D vector $R_{\text {com }}(x, y, z)$ follows the same time course as the 1-D displacement, $z$. All other parameters are the same as those defined in Eq. (4) above.

In this study, we have not monitored the tumor location as a function of time but have assumed that the tumor has a rectilinear trajectory following the time course described in Eq. (4). Although Eq. (4) may have limited applicability to individual patients, it has been found to provide a reasonable fit to population-based lung tumor motion. ${ }^{2,3}$ In particular, Seppenwoolde et al., ${ }^{2}$ who fluoroscopically imaged implanted markers for 20 lung cancer patients, found that Eq. (4) provided a reasonable fit of the breathing-induced tumor motion in these patients. Seppenwoolde et al. ${ }^{2}$ classified tumors according to those in the upper, middle and lower lobes of the lung and found that the degree of asymmetry of the breathing function (determined by the parameter $n$ in Eqs. (4), (5)) generally varied between $n=1$ and 3. Recently Engelsman et al. ${ }^{3}$ conducted a theoretical treatment planning study in which they assume that the lung tumor motion is described by Eq. (4) with a value of $n=3$. We include an analysis of the influence of the asymmetry of the breathing function on the dose distributions by conducting fluence convolved calculations with values of $n$ varying from 1 to 3 (see Figs. 1-3).

In the Monte Carlo implementation, $F_{\text {motion }}$ is divided into $m$ probability bins, from the position at exhale $\left(R_{0}\right)$ to the position at inhale $\left(R_{0}-a\right)$. The first $m-1$ bins are equally

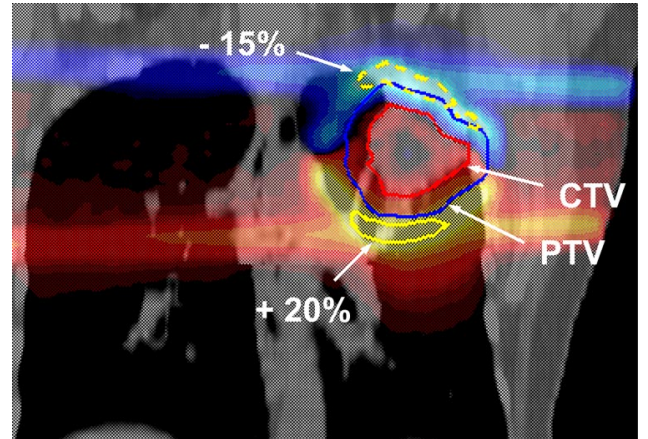

(a)

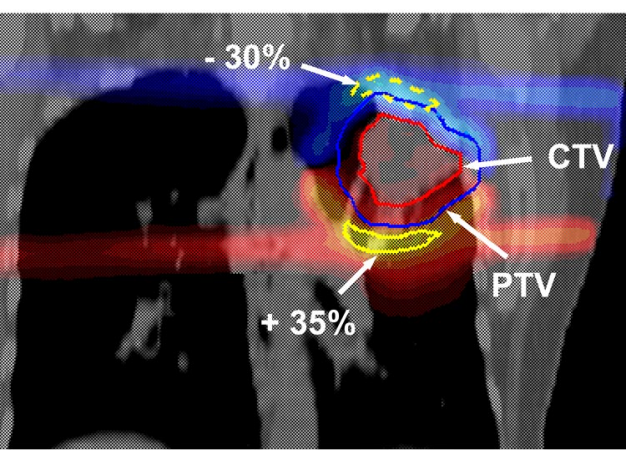

(b)

FIG. 2. Percentage dose difference maps (patient A) in the coronal view for (a) fluence convolution $(n=3)$-static dose, showing the $+20 \%$ and $-15 \%$ isodose lines; and (b) fluence convolution $(n=1)$-static dose, showing the $+35 \%$ and $-30 \%$ isodose lines. Nonhighlighted differences are within $\pm 2 \%$.

spaced-we use $3 \mathrm{~mm}$ bins to match the dose scoring voxel size - with the last bin being of arbitrary width in order to sample the correct $R_{\text {com }}$ amplitude. For example, if the amplitude of $R_{\text {com }}$ is $1.4 \mathrm{~cm}$ the first 4 bins would have width 3 $\mathrm{mm}$, and the last bin would have width $2 \mathrm{~mm}$. The position ( $x, y$ at a fixed $z$ location) and energy for each particle starting from the virtual source is determined by first sampling $\Phi_{\text {static }}$. The source particle's incident direction is determined from the position coordinates assuming that the particle originated from a point, ${ }^{13}$ i.e., $u=x / r^{\prime}, v=y / r^{\prime}, w$ $=z / r^{\prime}$, where $r^{\prime}=\left(x^{2}+y^{2}+z^{2}\right)^{0.5}$. To account for the GTVCOM motion in the $R_{\text {com }}$ direction, $F_{\text {motion }}$ is sampled to determine the positional translation, $\delta R_{\text {com }}$. The translations $\delta x, \delta y$, and $\delta z$ are determined according to the relations

$$
\begin{aligned}
& \delta x=\frac{\delta R_{\mathrm{com}}}{\left|R_{\mathrm{com}}\right|}\left(x_{I}-x_{E}\right), \quad \delta y=\frac{\delta R_{\mathrm{com}}}{\left|R_{\mathrm{com}}\right|}\left(y_{I}-y_{E}\right), \\
& \delta z=\frac{\delta R_{\mathrm{com}}}{\left|R_{\mathrm{com}}\right|}\left(z_{I}-z_{E}\right),
\end{aligned}
$$

where $\left|R_{\text {com }}\right|=\left(\left(x_{I}-x_{E}\right)^{2}+\left(y_{I}-y_{E}\right)^{2}+\left(z_{I}-z_{E}\right)^{2}\right)^{0.5}$. The following relation may then be used to describe the coordinate transformation from the static fluence distribution $\Phi_{\text {static }}$, in the unprimed coordinates, to the motion convolved fluence, $\Phi_{\text {motion }}$, in the primed coordinates:

$$
x^{\prime}=x-\delta x, \quad y^{\prime}=y-\delta y, \quad z^{\prime}=z-\delta z .
$$



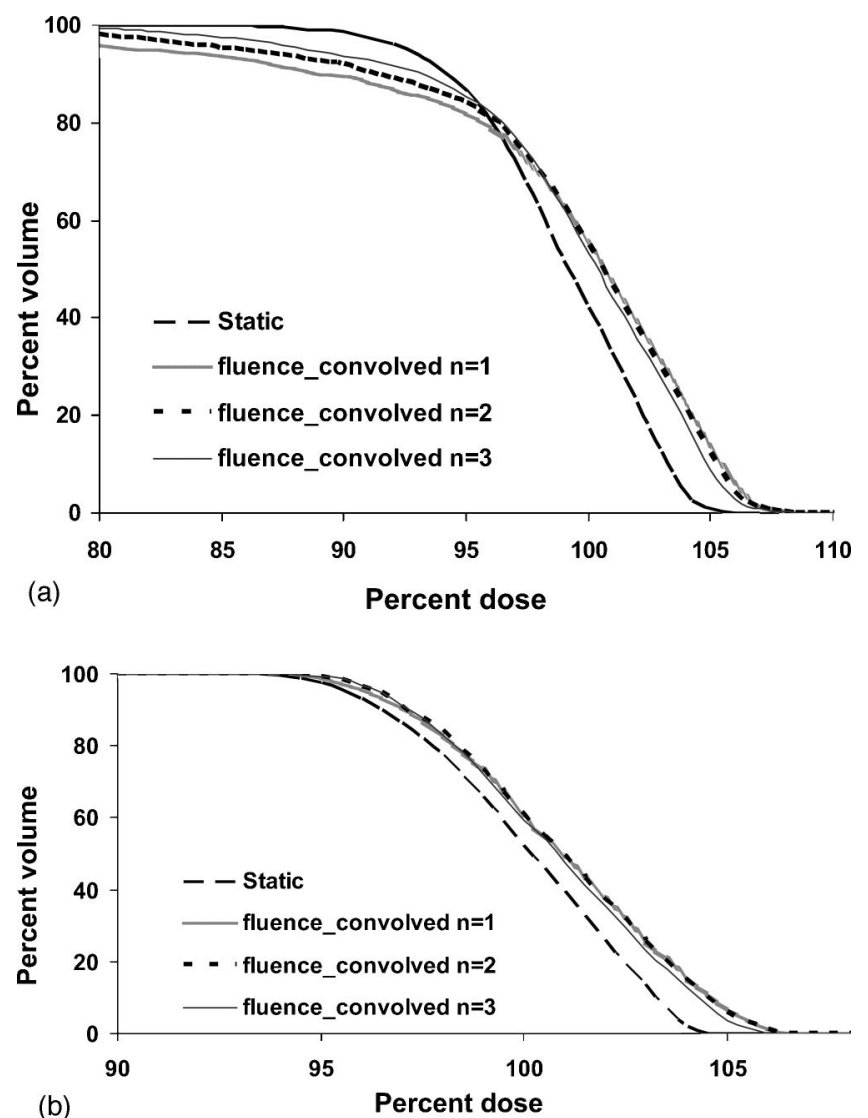

FIG. 3. Dose volume histograms (patient A) for (a) the PTV and (b) the CTV, for the static and fluence convolved treatment plans. The dose axis is scaled to emphasize the high gradient region of the DVH. Fluence convolved curves are included for breathing functions with varying degrees of asymmetry $(n=1-3)$.

The direction cosine vectors for each source particle are also appropriately modified as there is a change $r$, resulting from the translation

$$
u=\frac{x^{\prime}}{r^{\prime \prime}}, \quad v=\frac{y^{\prime}}{r^{\prime \prime}},
$$

and

$$
w=\frac{z^{\prime}}{r^{\prime \prime}} \quad \text { where } \quad r^{\prime \prime}=\sqrt{\left(x^{\prime 2}+y^{\prime 2}+z^{\prime 2}\right)} .
$$

\section{Dose convolution}

The influence of respiratory motion on the dose distribution was also evaluated using a dose convolution method, ${ }^{1}$ performed by convolving the static dose distribution, $D_{\text {static }}$, (calculated using $\Phi_{\text {static }}$ ) with the function, $F_{\text {motion, }}$ described above. The dose at a point $r$, that undergoes the motion, is calculated as follows:

$$
\begin{aligned}
D_{\text {motion }}(r) & =F_{\text {motion }} \otimes D_{\text {static }} \\
& =\int_{r^{\prime}} F_{\text {motion }}\left(r-r^{\prime}\right) D_{\text {static }}\left(r^{\prime}\right) d r^{\prime} .
\end{aligned}
$$

This implementation has been modified from the original version of Lujan et al. ${ }^{1}$ who only considered motion in the $z$-axis. $F_{\text {motion }}$ here is cast in the form of a 3 -D discrete matrix and the convolution with $D_{\text {static }}$ is conducted along the vector $R_{\text {com }}(x, y, z)$. As in the case with fluence convolution, the amplitude $\left|R_{\text {com }}\right|$ is divided into $3 \mathrm{~mm}$ equally spaced bins with the exception of the last bin which is of variable width.

\section{Monte Carlo treatment planning}

Treatment planning for three patients with tumors at different locations in the lung was conducted using the DPM Monte Carlo code within the framework of the UMPlan system. For all patients, static and fluence convolved planning was performed independently using the breath-hold exhale CT data sets. Dose convolved plans were generated by applying a post-processing convolution to the static beam dose distribution. The typical treatment plan beam configuration consisted of conformal 6/15 MV anterior, lateral and oblique fields, combined with segmental fields (directed from the same angles) to produce a dose distribution of $100 \pm 5 \%$ within the PTV. All plans were normalized to $100 \%$ at the isocenter.

DPM calculations were performed using a voxel size of $3 \times 3 \times 3 \mathrm{~mm}^{3}$, a $2 \mathrm{~mm}$ step size, and low energy electron and photon cut-off values of 200 and $50 \mathrm{keV}$, respectively. For each treatment plan, approximately 1 billion histories per field were simulated, resulting in $1 \sigma$ statistics of roughly less than $1.5 \%$ in the calculated dose, if we include the $1 \%$ latent uncertainty in the reconstructed fluence (from the virtual source). The time required for these simulations was approximately 8 hours per 1 billion particles, running on a single $1 \mathrm{GHz}$, VMS-based, Alpha processor.

\section{RESULTS AND DISCUSSION}

\section{A. Influence of the breathing function asymmetry}

Figure 1 shows plots of the probability as a function of GTV-COM position $(r)$, as described by Eq. (5), for different values of $n(n=1,2,3)$. The probability of finding the GTV-COM at exhale increases as the value of $n$ increases indicating that the breathing PDF becomes asymmetrically weighted toward the exhale position with increasing $n .{ }^{18}$ Figure 2 shows difference maps between the fluence convolved and static dose distributions (i.e., fluence-static) in the coronal view for patient A. The fluence convolved calculations were performed by sampling breathing PDF's [Eq. (5)] for two different values of $n(n=1,3)$. An increase in the dose differences (fluence-static) is noted as the value of $n$ decreases; the maximum differences are approximately $\pm 20 \%$ for $n=3$, $\pm 30 \%$ for $n=2$ and $\pm 40 \%$ for $n=1$. This trend is expected if we consider that the static treatment plans were performed using the exhale CT scan-as $n$ decreases the breathing function becomes more symmetric with the result that a greater fraction of time is spent at the inhale position relative to larger values of $n$. Figure 3 illustrates the dose-volume-histogram (DVH) for the static and fluence convolved plans (with $n=1-3$ ), shown specifically for the PTV [Fig. 3(a)] and the CTV [Fig. 3(b)]. We see that the 
TABLE I. Amplitudes of the GTV center-of-mass excursion between the breath-hold exhale and inhale CT phases. Values along the independent axes represent the absolute differences between the exhale and inhale positions. The direction of motion along each of the patient axes is provided in parentheses. Also shown are the magnitudes of the $R_{\text {com }}(x, y, z)$ vector. Positive movements from exhale to inhale are directed along the patient's: left $(+x)$, posterior $(+y)$, and superior $(+z)$.

\begin{tabular}{cccc}
\hline \hline & Patient $\boldsymbol{A}$ & Patient $\boldsymbol{B}$ & Patient $\boldsymbol{C}$ \\
\hline $\boldsymbol{x}(\mathbf{c m})$ & 0.17 (lt.) & 0.11 (lt.) & 0.21 (rt.) \\
$\boldsymbol{y}(\mathbf{c m})$ & 0.64 (ant.) & 0.24 (ant.) & 1.28 (ant.) \\
$\boldsymbol{z}(\mathbf{c m})$ & 1.26 (sup.) & 0.96 (inf.) & 0.09 (sup.) \\
$\boldsymbol{R}_{\text {com }(\mathbf{c m})}$ & 1.42 & 1.00 & 1.30 \\
\hline \hline
\end{tabular}

gradient of the PTV DVH is reduced as the value of $n$ decreases - the DVH shoulder is degraded while the high dose region is increased showing the influence of the cold and hot spots which worsen as $n$ decreases. A similar trend was found for the CTV DVH's [Fig. 3(b)], however, the differences were less significant in comparison to the PTV as a consequence of proper PTV design in the static plan.

\section{B. Treatment planning analysis (static vs motion convolved planning)}

Amplitudes for the exhale/inhale excursion of the GTV$\mathrm{COM}$ are presented in Table I for the 3 patients planned in this study. For patient A, the GTV-COM is found to move $1.3 \mathrm{~cm}$ in the superior direction and $0.6 \mathrm{~cm}$ anteriorly. The largest motion for patient $\mathrm{B}$ was observed in the inferior direction $(\sim 1 \mathrm{~cm})$ and for patient $\mathrm{C}$ this occurred in the anterior direction $(\sim 1 \mathrm{~cm})$.

Illustrated in Fig. 4 are the dose difference maps in the coronal view (for patient A) for (a) dose convolution ( $n$ $=3)$-static, and (b) fluence $(n=3)$-dose $(n=3)$. Note that the difference map for the fluence convolution $(n=3)$ and static dose distributions is presented in Fig. 2(a). In the difference maps shown in Figs. 2(a) and 4(a), hot and cold spots are located superiorly and inferiorly, respectively. This is because the static treatment plans were conducted in the exhale position and that the GTV-COM for this patient moved predominantly in the superior direction during inhale; that is, relative to the exhale position, the superior region of the target moves out of the beam during inhale while the inferior edge moves into the beam resulting in the respective cold and hot spots. From the difference maps in Figs. 2(a) and 4(a) we also see that the fluence and dose convolved distributions are generally in good qualitative agreement, however, a more detailed evaluation reveals some differences. In the fluence convolved case [Fig. 2(a)] the maximum and minimum differences are $+25 \%$ and $-20 \%$, respectively - these differences are not symmetric as there is preferentially more dose deposited in the lung due to the increased lateral electron transport in this region. This illustrates that fluence convolution is able to account for the variation in tissue densities surrounding the target. For the dose convolution situation [Fig. 4(a)] the maximum and minimum differences are symmetric $( \pm 25 \%)$ as the convolution is spatially invariant, showing that this method does

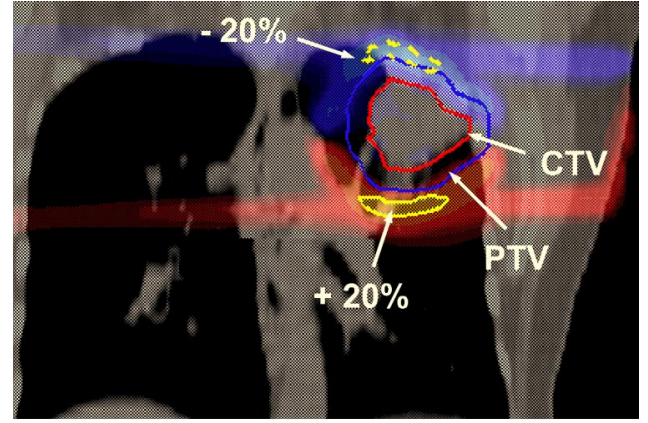

(a)

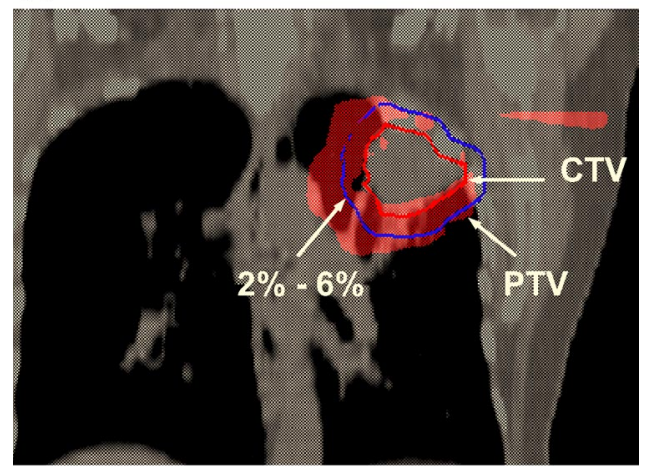

(b)

FIG. 4. Percentage dose difference maps (patient A) in the coronal view for (a) dose convolution $(n=3)$-static dose, showing the $\pm 20 \%$ isodose lines; and (b) fluence convolution $(n=3)$-dose convolution $(n=3)$, highlighting the +2 to $+6 \%$ dose difference region. Nonhighlighted differences are within $\pm 2 \%$.

not account for the influence of inhomogeneous tissues on the dose distribution. The differences between fluence and dose convolution are further demonstrated in Fig. 4(b) (fluence-dose convolution) where we find positive differences up to 7\%. Figure 5 illustrates the DVH's for patient A for (a) the PTV (differential) and (b) the CTV (integral). Plots are shown for the static and the fluence and dose convolved dose distributions. It is clear that there is a systematic shift toward higher doses for the fluence convolved differential DVH [Fig. 5(a)] relative to the static and dose convolved cases. The reason for this is that, while hot and cold spots are found at the inferior and superior edges of the PTV, respectively, the hot spots are greater and tend to dominate the overall dose to the PTV. For the dose convolution plan the PTV DVH more closely agrees with that of the static case because the hot and cold spots tend to offset each other. CTV differences between the static, fluence and dose convolved plans are similar in trend to that of the PTV but are smaller in magnitude as observed in Fig. 5(b).

Dose difference maps and DVH's for the motion convolved and static beam calculations for patient B are illustrated in Fig. 6. The beam arrangement for patient B was similar to that of patient A, however, patient B differed with respect to (a) the location of the hot and cold spots, and (b) the location of the tumor. Figure 6(a) illustrates a difference 


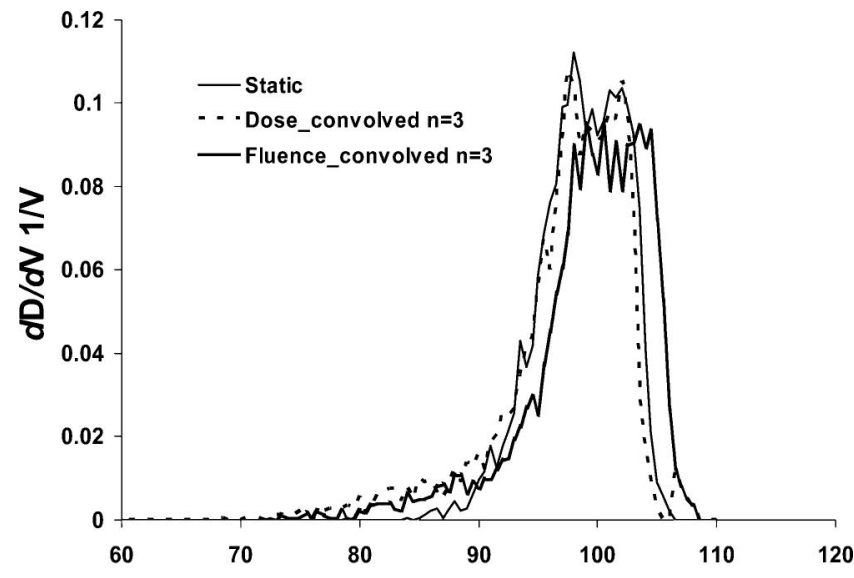

(a)

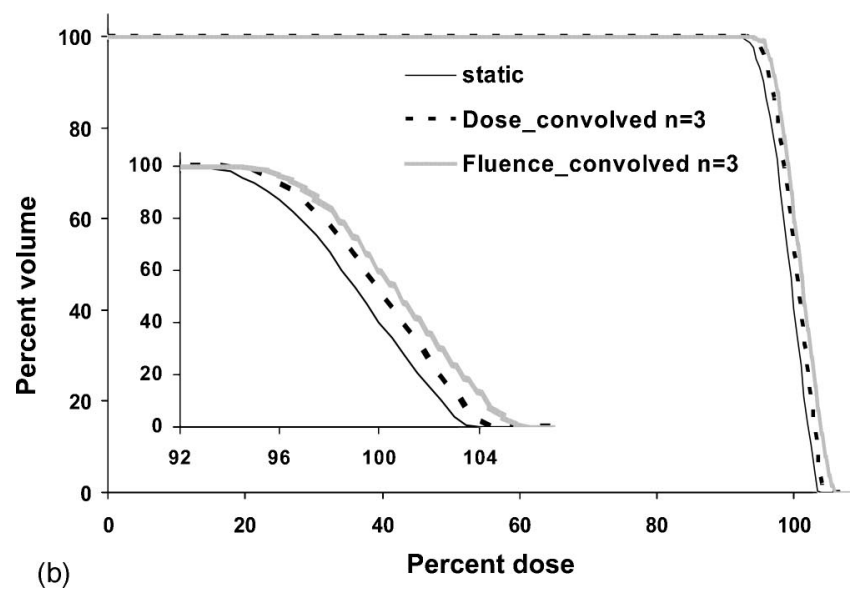

FIG. 5. Dose volume histograms (patient A) for the static, fluence and dose convolved treatment plans for (a) the PTV (differential DVH) and (b) the CTV. Fluence and dose convolved calculations were performed assuming a breathing function with an $n=3$ degree of asymmetry. The inset in (b) highlights the high gradient region of the DVH.

map between the fluence convolved $(n=3)$ and static doses in the sagittal view, where the maximum and minimum dose differences are $+28 \%$ and $-24 \%$, respectively. These differences are larger than those for patient A because the GTV for patient $\mathrm{B}$ is surrounded by much more lung tissue. In contrast to patient $\mathrm{A}$, the hot and cold spots in the difference maps for patient B are located superiorly and inferiorly respectively; in the case of patient B, the GTV-COM moves inferiorly during inhale resulting in a cold spot as the inferior edge moves out of the beam, and a hot spot as the superior edge moves into the beam. An analysis of the dose difference map between the fluence and dose convolved distributions showed maximum differences of $+5 \%$. Much like the case of patient $\mathrm{A}$, these differences were predominantly positive and resulted from the shift invariance assumption of dose convolution in the presence of inhomogeneous tissues. DVH's for the PTV for patient B are shown in Fig. 6(b). A subtle dose reduction at the shoulder of the PTV DVH is noted for the motion (fluence and dose) convolved plans, however, the differences relative to the static case are fairly insignificant. A likely reason for this is that the Monte Carlo static dose calculation is expected to correctly account for

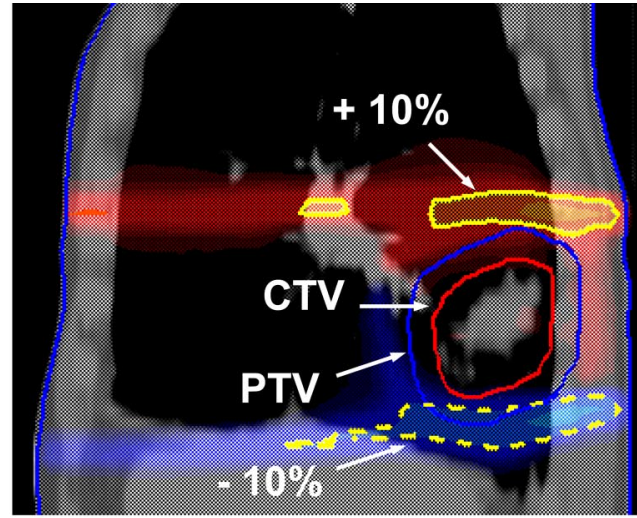

(a)

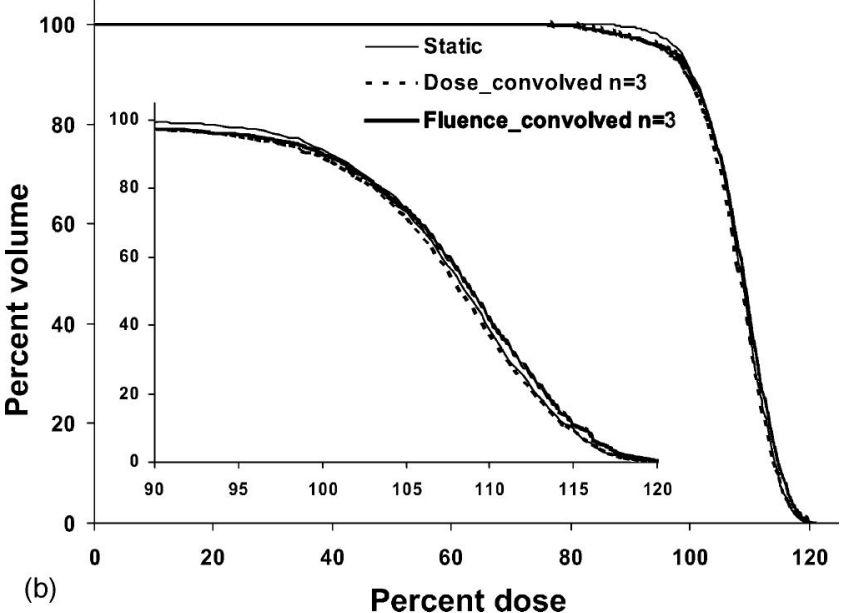

FIG. 6. Percentage dose difference map (patient B) in the sagittal view for fluence convolution $(n=3)$-static dose, showing the $\pm 10 \%$ isodose lines. (b) shows dose volume histograms for the PTV. Included are plots for the static, fluence and dose convolved treatment plans. The inset highlights the high gradient region of the DVH.

the spread of dose from the target into the surrounding lung; convolving the static dose in this region either by fluence or dose convolution is therefore unlikely to cause a substantial variance in the dose distribution relative to the static case. This effect has been described previously. ${ }^{11}$ Similarly, differences in the CTV DVH's between static and motion convolved plans were minimal suggesting that the PTV was relatively well designed, i.e., the dose to the CTV was not compromised in the presence of motion.

Presented in Fig. 7(a) is the fluence-static dose difference display for the 7-field conformal beam arrangement for patient $\mathrm{C}$. In this case, the largest differences $(+14 \%$ and $-11 \%$ ) occur in the AP direction-this is expected because the GTV-COM moves predominantly in the anterior direction (see Table I). The difference map between the fluence and dose convolved plans is shown in Fig. 7(b). We see differences of $+4 \%$ in the vicinity of the PTV, however, much larger differences are noted at the patient surfaces, $-50 \%$ and $+20 \%$ at the anterior and posterior surfaces, respectively. As the patient moves posteriorly, the hot spot is found at the anterior surface. This surface dose effect has been previously described by Craig et al., ${ }^{8}$ and occurs be- 


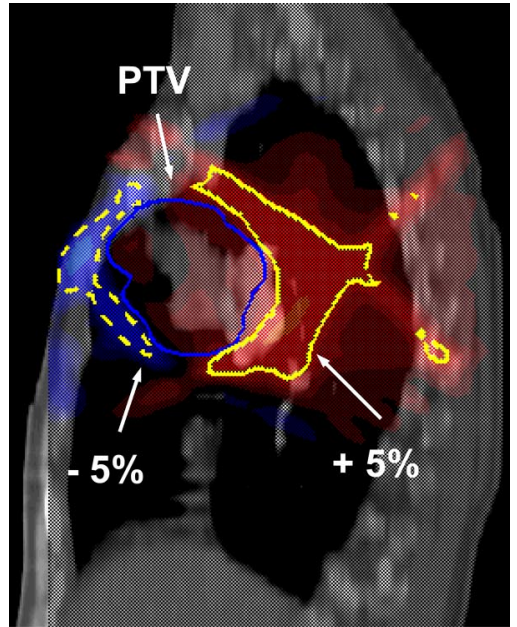

(a)

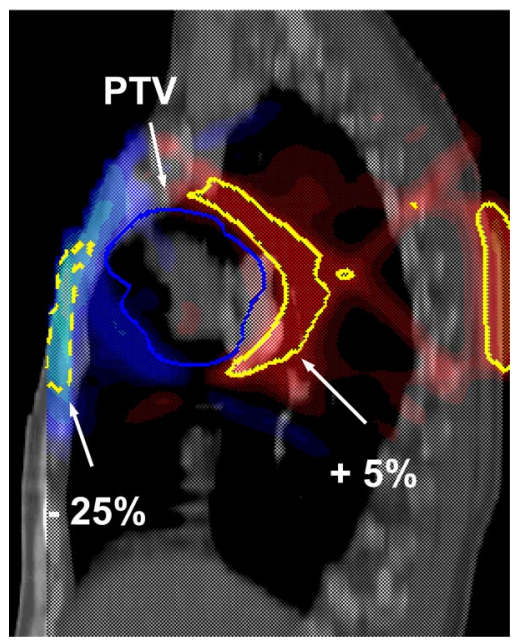

(b)

FIG. 7. Percentage dose difference maps (patient $\mathrm{C}$ ) in the sagittal view for (a) fluence convolution $(n=3)$-static dose showing the $\pm 5 \%$ isodose lines; and (b) fluence convolution $(n=3)$-dose convolution $(n=3)$ showing the $+5 \%$ and $-25 \%$ isodose lines.

cause the dose convolution method represents a shifting of the dose distribution. Outside the patient, the dose is zero with the result that there is no dose to "shift" into the patient. $^{8}$ Note that the surface dose limitation of dose convolution was observed for the other patients in this study as well. Unlike in the work by Craig et al., ${ }^{8}$ we have not modified the dose convolution method to correct for the surface dose irregularities. DVH's for the CTV and PTV for patient $\mathrm{C}$ were found to follow a different pattern compared to the other two patients: fluence convolution predicts more dose to both the CTV and PTV. One of the reasons for this is that the tumor motion occurs mostly in the anterior-posterior direction, which coincides with the direction of some of the beams used for the treatment plan. The decreased SSD in the fluence motion compensated plan leads to an increased dose to the PTV. This effect is not properly accounted for in the dose convolved plan where the dose is precalculated at the static SSD.

\section{Beam edge vs inhomogeneity effects}

In addition to hot/cold spots in the vicinity of the PTV, the dose difference maps in the previous analyses (Figs. 2, 4, 6-7) show differences along the beam edges. These differences are a result of the convolution of the static dose distribution which tends to blur out the beam penumbra causing an unsharp edge. In the case of fluence convolution, the dose distribution is also influenced by the local tissue densitiesthe dose distribution will be spatially variant in the presence of inhomogeneous tissues. To better understand the beam edge versus inhomogeneity effect on the motion convolved dose distributions we performed a calculation for patient $\mathrm{B}$ assuming that the CT densities were water equivalent (all values were set to 1.0). The idea was to isolate the beam edge differences from those due to the tissue inhomogeneities. Results of this analysis showed differences of up to $+23 \%$ due only to the beam edge effect of the fluence convolution. The combined dose difference map (fluence convolution-static) in the heterogeneous density case [shown earlier in Fig. 6(a)] includes both the beam edge and inhomogeneity effects and shows maximum differences of $+28 \%$. The spatial variation of the dose distribution due to the inhomogeneity effect, for this particular plan, was on the order of $5 \%$, which is consistent with the differences noted between fluence and dose convolution for this same case.

\section{CONCLUSION}

In this study we account for some of the effects of breathing-induced target motion in the vicinity of the lung using convolution methods. Significant hot and cold spots $( \pm 15 \%-25 \%)$ were found in the dose difference maps between the motion convolved and static dose distributions as a result of the target motion. Smaller differences were observed between fluence and dose convolution-these differences are mainly due to the spatial invariance of the dose convolution distribution in the presence of the low density lung tissue. This investigation would not be complete without addressing the limitations of convolution methods in accounting for respiratory induced motion. Both fluence and dose convolution methods do not address the dose per fraction effects, which may potentially be important as recently demonstrated in a study by Craig et al. ${ }^{9}$ In addition, dose convolution assumes shift invariance of the dose distribution, which is shown both here and in a recent study by Craig et $a l .{ }^{8}$ to have limitations. Finally, we have not included in this study an evaluation of the doses to the normal lung because convolution methods do not take into account the increase in volume of the lung with inhalation. The change in volume as well as the deformation of the normal lung tissue during respiration ${ }^{2}$ must be correctly accounted for in order to accurately estimate the dose to the lung. Some work ${ }^{19,20}$ has begun in this area but further investigation is clearly warranted. 


\section{ACKNOWLEDGMENTS}

This work has been supported in part by NIH Grant P01CA59827 and by a University of Michigan Cancer Center grant funded by the John and Suzanne Munn Endowment.

${ }^{a)}$ Electronic mail: indrin@med.umich.edu

${ }^{1}$ A. E. Lujan et al., "A method for incorporating organ motion due to breathing into 3D dose calculations," Med. Phys. 26, 715-720 (1999).

${ }^{2}$ Y. Seppenwoolde et al., "Precise and real-time measurement of 3D tumor motion in lung due to breathing and heartbeat, measured during radiotherapy," Int. J. Radiat. Oncol., Biol., Phys. 53, 822-834 (2002).

${ }^{3}$ M. Engelsman et al., "The theoretical benefit of beam fringe compensation and field size reduction for iso-normal tissue complication probability dose escalation in radiotherapy of lung cancer," Med. Phys. 30, 1086-1095 (2003).

${ }^{4}$ M. Rosu et al., "Alterations in normal liver doses due to patient motion," Int. J. Radiat. Oncol., Biol., Phys. 57, 1472-1479 (2003).

${ }^{5}$ A. E. Lujan et al., "A method for incorporating organ motion due to breathing into 3D dose calculations," Med. Phys. 26, 715-720 (1999).

${ }^{6}$ G. S. Mageras et al., "A method of incorporating organ motion uncertainties into three dimensional conformal treatment plans," Int. J. Biol. Phys. 35, 335-342 (1996).

${ }^{7}$ S. D. McCarter et al., "Evaluation of the validity of a convolution method for incorporating tumour movement and set-up variations into the radiotherapy treatment planning system," Phys. Med. Biol. 45, 923-931 (2000).

${ }^{8}$ T. Craig et al., "Limitations of a convolution method for modeling geometric uncertainties in radiation therapy. I. The effect of shift invariance," Med. Phys. 30, 2001-2011 (2003).

${ }^{9}$ T. Craig et al., "Limitations of a convolution method for modeling geo- metric uncertainties in radiation therapy. II. The effect of a finite number of fractions," Med. Phys. 30, 2012-2020 (2003).

${ }^{10}$ W. A. Beckham et al., "A fluence-convolution method to calculate radiation therapy dose distributions that incorporate random set-up error," Phys. Med. Biol. 47, 715-720 (1999).

${ }^{11}$ I. J. Chetty et al., "A fluence convolution method to account for respiratory motion in three-dimensional dose calculations of the liver: A Monte Carlo study," Med. Phys. 30, 1776-1780 (2003).

${ }^{12}$ ICRU Report 50, International Commission on Radiation Units and Measurements: Prescribing, recording and reporting photon beam therapy, Bethesda, Maryland, 1993.

${ }^{13}$ I. J. Chetty et al., "A virtual source model for Monte Carlo modeling of arbitrary intensity distributions," Med. Phys. 27, 166-172 (2000).

${ }^{14}$ I. J. Chetty et al., "Photon beam relative dose validation of the DPM Monte Carlo code in lung-equivalent media," Med. Phys. 30, 563-573 (2003).

${ }^{15}$ J. Sempau et al., "DPM, a fast, accurate Monte Carlo code optimized for photon and electron radiotherapy treatment planning calculations," Phys. Med. Biol. 45, 2263-2291 (2000).

${ }^{16} \mathrm{~J}$. M. Balter et al., "Improvement of CT-based treatment planning models of abdominal targets using static exhale imaging," Inst. Phys. Conf. Ser. 41, 939-943 (1998).

${ }^{17} \mathrm{~S}$. Shimizu et al., "Detection of lung tumor movement in real-time tumor-tracking radiotherapy," Int. J. Radiat. Oncol., Biol., Phys. 51, 304-310 (2001).

${ }^{18}$ A. E. Lujan et al., "A method for incorporating organ motion due to breathing into 3D dose calculations in the liver: Sensitivity to variations in motion," Med. Phys. 30, 2643-2649 (2003).

${ }^{19}$ M. Ding et al., "Dose correlation for thoracic motion in radiation therapy of breast cancer," Med. Phys. 30, 2520-2529 (2003).

${ }^{20} \mathrm{~B}$. Schaly et al., "Accounting for variable anatomy in radiation therapy dose distributions," Med. Phys. 30, 1428 (2003) (abstract). 